\title{
Traceable measurements by atomic spectrometry
}

\author{
Mike Sargent \\ Metrology Consultant, Surbiton, UK. metrology@misar.uk; (iD) https://orcid.org/0000-0001-6325-7944
}

The concept of traceable measurements is simple and well established.' Traceability solves the problem of ensuring that measurements of the same quantity are comparable even when made by different people, in different places or at different moments in time. Almost all measurements require a calibration of some kind. If measurements of the same quantity always use calibration standards derived ultimately from the same unique reference point, or primary standard, all will be based on the same measurement unit and hence comparable with each other. It is rarely possible to calibrate all measurements directly against the unique primary standard. The answer is a traceability "chain" comprising a series of calibrations using intermediate reference standards, the values and uncertainties of which are passed to the next link in the chain.

Provided that the measurement procedure is relatively straightforward, such as with everyday measurements of length and mass, comparability is sufficient to ensure consistent (or equivalent) measurement results. However, measurements such as amount of a chemical substance increase the complexity of the measurement procedure. In this

\section{DOI: $10.1255 /$ sew.2021.a 1}

(c) 2021 The Author

Published under a Creative Commons BY-NC-ND licence

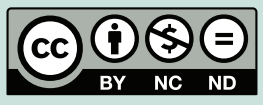

case traceable calibration values will probably not be adequate on their own to achieve consistent results. Additional precautions such as formal method validation are then necessary. This applies to most measurements made by analytical chemists and is discussed later.

\section{Global traceability}

For a long time each community requiring traceable measurement results devised its own units and primary standards as well as the means to disseminate them. However, the $19^{\text {th }}$ Century industrial revolution created the need for a single, global system. A convention held in Paris in 1875 resulted in an international treaty known as the Metre Convention. ${ }^{2}$ The structure envisaged in the Metre Convention to support traceable measurements has continued into the $21^{\text {st }}$ Century. Originally there were just three base units-for mass, length and time. Further base units were subsequently added and there are presently seven as shown in Table 1. These form the basis of the International System of Units, the SI. ${ }^{3}$ More than seven units are in common use, but the others can be directly derived from those in the table. The most recent addition, in 1971, was the mole which is the base unit for the amount of a chemical substance. Until that point the Metre Convention was concerned solely with physical measurements.

\section{Traceability in chemistry Nevertheless, analytical chemists have become accustomed to using the phys- ical measurement system because it underpins traceable calibrations for balances and volumetric glassware. This is important, but not an end in itself, because analytical chemistry is about more than determination of mass and volume per se; the analyst must meas- ure the amount of the required element or compound. To achieve this, most instrumental methods require calibration of the instrument using solutions or solid artefacts containing known amounts of the target analyte. With direct analysis of solid samples the instrument response varies substantially with the overall composition. In this case reliable calibra- tion requires the use of known values from "previously analysed materials" of very similar composition to the sample, i.e. matrix-matched reference materi- als. Both these and calibration solu- tions are chemical analysis standards}

Table 1. The base units of the International System of Units (SI).

\begin{tabular}{|l|c|c|}
\hline Physical quantity & Name of unit & Abbreviation \\
\hline Mass & kilogram & $\mathrm{kg}$ \\
\hline Length & metre & $\mathrm{m}$ \\
\hline Time & second & $\mathrm{S}$ \\
\hline Temperature & kelvin & $\mathrm{K}$ \\
\hline Amount of substance & mole & $\mathrm{mol}$ \\
\hline Electric current & ampere & $\mathrm{A}$ \\
\hline Luminous intensity & candela & $\mathrm{cd}$ \\
\hline
\end{tabular}


available from a wide variety of suppliers. However, for much of the $20^{\text {th }}$ Century no attempt was made to apply the concept of traceability to ensure the consistency of their values. This was not feasible at the time due to the lack of a global chemical measurement infrastructure comparable to the physical one.

In the 1980s a move began to address numerous quality issues identified with chemical measurements. In particular, measurement laboratories were encouraged to seek accreditation to the ISO/IEC 17025 or ISO 15189 standards. Subsequently, this was extended to include accreditation of reference material producers to ISO 17034. Meeting the requirements of these standards for traceable chemical measurement results necessitated the creation of an appropriate international infrastructure. This was done by extending the one which had been developed for physical measurements over more than 100 years. ${ }^{4}$ Calibrants with traceable values are now widely available, including many needed for applications of atomic spectrometry.

Making use of these materials requires laboratories to become the last link in the traceability chain. In addition to the chemical calibration standard or solution there will be another chain for calibration of the balance and perhaps also for volumetric glassware. Whether or not calibration values must be traceable depends on whether they contribute significantly to the overall uncertainty of the measurement result. Hence, matrix materials used for method validation should have traceable values. This is obvious if a matrix correction or recovery factor is subsequently applied to the results. However, even when a certified reference material (CRM) is used only to confirm $100 \%$ recovery of analyte, the uncertainty of the certified value(s) still contributes to the overall uncertainty estimation for the measurement results.

\section{Traceability in the laboratory}

The tasks needed by the laboratory to take its place at the end of the traceability chain are straightforward, because they comprise best practice specified for laboratory accreditation. In summary, properly validate measurement procedures, estimate uncertainty and ensure full documentation. Calibration standards or matrix reference materials used in the procedure should have a traceability chain which is complete and fully documented. Commercial availability of suitable materials is generally good for many applications of atomic spectrometry. This is particularly true for most elemental analysis applications using techniques such as atomic absorption spectrometry, inductively coupled plasma-optical emission spectroscopy and inductively coupled plasma-mass spectrometry (ICP-MS) which are calibrated with single or multi-element solutions. Solid sampling techniques such as X-ray fluorescence or arc/spark optical emission spectroscopy are more difficult, due to the need for matrix-matched calibrations. However, older CRM stocks for applications such as steels, alloys and ores are steadily being replaced with modern materials having traceable values.

Wherever possible purchase commercial reference materials from a supplier accredited in accordance with ISO 17034. However, it is still prudent to carefully assess the materials. For example, ISO 17034 allows non-certified values to be included on a certificate. This is useful, but such values are unsuitable for calibration purposes and are unlikely to be covered by the certificate's traceability statement. There is no standard format for traceability statements and many are limited to unhelpful phrases such as "traceable to the $\mathrm{SI}^{\prime}{ }^{5}$ Users should look for more detail, either in a more comprehensive statement or as additional information supplied with it. This usually includes the source of the traceable calibration values used in assigning values to the material.

Assignment of values to matrix reference materials often requires complicated methods and the producer should provide information about these, including how they were validated. Matrix reference materials values are frequently assigned by inter-laboratory comparisons. Claims such as "traceable to the methods used by the participants" are

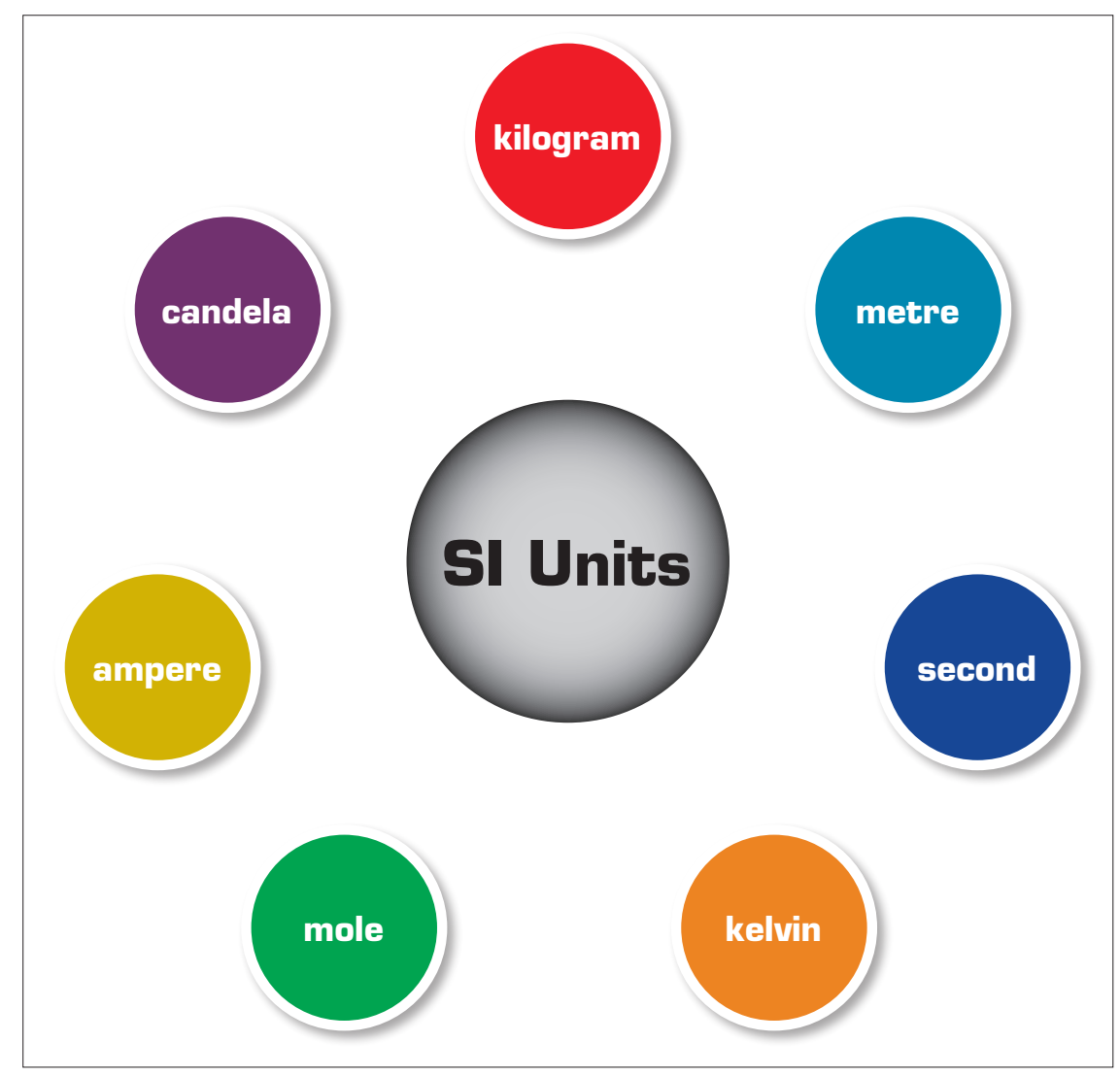


unhelpful, so look for additional information. Materials with a very long shelf life, such as those used in metallurgy and geochemistry, also need caution. Many still on sale today were certified before current best practice using informal interlaboratory exercises, often without traceable calibrants. Moreover, these older materials may sometimes be used as calibration standards for certification of modern reference materials.

\section{Traceability in practice}

Sometimes traceable elemental calibration solutions are not available commercially or laboratories are not in a position to purchase them. Commercial solutions of analytes such as organo-metallic or isotopic materials are also less common. The laboratory then needs to prepare them in-house and assign traceable values. In principle this is not difficult and was once the norm for analytical laboratories. However, the devil lies in the detail. A common approach is to take a known mass of the analyte from a sample having known purity and dissolve it in a known mass or volume of solvent. Provided the balance and volumetric glassware have traceable calibration values the laboratory can demonstrate the traceability of the assigned value. The devil is the purity of the solid analyte sample. It does not necessarily need to be ultra pure, but the value assigned to the purity must be reliable and traceable. Ideally, the pure material should be a CRM but these are often unavailable. It is tempting to use commercial reagent grade materials instead, but these usually come with only an approximate estimate of purity or a batch specification. Such values are inappropriate for the preparation of calibration solutions. Some possible alternatives for three application areas are discussed below.

\section{Elemental analysis}

Unfortunately, few pure elements for preparation of calibration solutions are available as CRMs and undertaking a full assay procedure is not a trivial task. ${ }^{6}$ However, many metals are widely available for other purposes in very high purity forms. These may be used provided suitable precautions are taken. A routine spectroscopic scan will confirm the absence of other elements even at trace levels. If impurities are identified at significant concentrations, it may be easier to find an alternative source rather than attempt to measure their amount accurately in order to apply a correction. In many cases, metals sold as " 4 or 5 nines pure" are suitable but there are two potential problems. These metals may have pockets of occluded gases which could introduce an error in the calculated concentration of the solution. Some metals are prone to oxidation, producing a surface oxide layer and the surface should be cleaned before weighing them. Both errors can be reduced by using rod or wire instead of turnings or fine powders. It is also essential to ensure that metal is taken into solution quantitatively, which may not always be a trivial task.

Solutions of metals such as rhodium, which are available in high purity form but are difficult to dissolve, or of nonmetallic elements, may be prepared from a suitable salt. However, dissolution of a known weight of the salt is rarely sufficiently accurate due to the lack of reliable data on stoichiometry, water content or impurities. The preferred option is to assay a solution of approximate concentration using a technique such as EDTA titrimetry. EDTA solutions with certified concentration are readily available or can be assayed using a certified solution of a metal such as silver. To ensure traceable results, each such calibration step must be accurate and its measurement uncertainty documented.

\section{Speciation analysis}

The hyphenation of ICP-MS with liquid or gas chromatography, or field-flowfractionation allows analysis of organic compounds containing a metal atom or heteroatoms such as $\mathrm{P}$ and $\mathrm{S}$. Used with appropriate analytes, this offers greater sensitivity than most conventional organic mass spectrometers and selectivity for molecules containing the target atom. It also allows an easier route to traceable measurement results than organic mass spectrometry. The latter also uses calibration solutions prepared by weighing analyte samples of known purity but there is a problem. Organic compounds close to $100 \%$ pure are rarely available, so an accurate purity assessment is essential to avoid significant errors. This is not a trivial task ${ }^{7}$ and presents major difficulties with large molecules.

There is, however, an alternative calibration approach for speciation applications using hyphenated ICP-MS. As the instrument response corresponds to the total amount of the metal or heteroatom, it can be calibrated using an inorganic solution of the target atom. Calibration solutions with traceable values are readily purchased or prepared as described for elemental analysis. This measurement does, of course, determine the total amount of the element and not the target organic molecule. However, the element-specific chromatogram will reveal the presence of any impurity peak(s) containing the target atom so a traceable value for the target molecule can be calculated. This approach has been used for compounds such as selenoproteins, ${ }^{8}$ ferritin-bound iron ${ }^{9}$ and carboplatin adducts. ${ }^{10}$

\section{Isotopic analysis}

It is sometimes forgotten that ICP-MS always makes isotopic measurements. A specific isotope of the target element is compared between the sample and calibrant solutions. This works provided the isotope's abundance is the same for both. Unless the element is mono isotopic there is no guarantee that this is true. For example, the natural isotopic abundance of lead varies quite widely and isotopic fractionation of some elements can be caused by processing techniques. Hence, to obtain traceable results it is necessary to determine the isotopic abundance of both the calibrant and the sample, applying a correction if necessary.

Calibration of mass spectrometry using isotope dilution analysis (IDMS) requires addition to the sample of an isotopically labelled material (the "spike"). This comprises, or is labelled with, an isotope having low abundance in the natural sample. Its ratio to a naturally abundant isotope is then determined. The popularity of IDMS calibration has increased 
with the wide use of ICP-MS, ${ }^{11}$ but it is then essential to compensate for a variation in instrument response between the two isotopes, the so-called mass bias correction. Traceable values can be achieved by obtaining or preparing solutions of the isotopic spike with known amounts of the chosen isotope. An alternative approach is known as the "double" or "reverse" IDMS technique ${ }^{12}$ which uses a calibration solution of the natural analyte. This requires a relatively complicated measurement procedure involving a sequence of "blends" in which both the sample and calibration solutions are spiked with the isotopic material.

\section{Where next?}

As we have seen, traceable results have a long and successful history with physical measurements. Fortunately, the global measurement infrastructure has now been extended to support traceable values in analytical chemistry. Hence, any spectrometry laboratory should be able to provide traceable measurement results by adopting current best practice. However, this depends on the availability of calibration standards and matrix reference materials with traceable values. Supply is already good for many elemental calibration solutions, but there is much more to do with matrix-matched standards. These are essential, for example, with the solid sampling techniques used in metallurgy and geochemistry. On the positive side, developing the chemical measurement infrastructure has encouraged many more national institutes to produce chemical standards and CRMs. These are available to laboratories but also provide the means for commercial producers to establish traceable values for their own products. Many of the national institutes also have active research programmes addressing traceability issues, including novel applications such as organo-metallics, protein analysis and isotopic measurements. Thus, we can be assured that traceable measurements in atomic spectrometry will have a long and successful future.

\section{References}

1. M. Sargent, "Quality assurance: Traceability", Encyclopedia of Analytical Science, $3^{\text {rd }}$ Edn. Elsevier, Vol. 8, pp. 527-535 (2019). https:// doi.org/10.1016/B978-0-12-4095472.14398-7

2. BIPM, The Metre Convention. https://www.bipm.org/en/worldwide-metrology/metre-convention/

3. NPL, The International System of Units (SI). https://www.npl.co.uk/ si-units

4. BIPM, Consultative Committee for Amount of Substance: Metrology in Chemistry and Biology (CCQM). https://www.bipm.org/en/committees/cc/ccam/

5. M. Sargent, "The provision and use of traceability statements for reference materials", Accred. Qual. Assur. 25, 367-372 (2020). https://doi. org/10.1007/s00769-020-01450-8

6. J. Vogl, The IAWG Roadmap for the Purity Determination of Pure Metallic Elements. BIPM, CCQM-IAWG/17-28 (2017). https://www.bipm.org/wg/ CCQM/IAWG/Allowed/April_2017/ CCQM-IAWG 17-28.pdf

7. K.A. Lippa, D.L. Duewer, M.A. Nelson, S.R. Davies and L.G. Mackay, "The role of the CCQM OAWG in providing SI traceable calibrators for organic

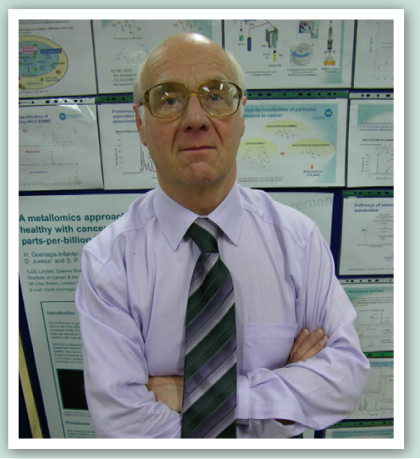

Dr Mike Sargent is an analytical chemist with over 50 years experience of atomic and molecular spectrometry, reference materials production, analytical quality assurance, laboratory management and chemical metrology. He played a leading role in developing UK and international chemical metrology and chairs or participates in a number of committees for analytical chemistry and reference material production. chemical measurements", Accred. Qual. Assur. 24, 407-415 (2019). https://doi.org/10.1007/s00769019-01407-6

8. M.E. del Castillo Busto, C. Oster, S. Cuello-Nuñez, C.L. Deitrich, A. Raab, A. Konopka, W.D. Lehmann, $\mathrm{H}$. Goenaga-Infante and P. Fisicaro, "Accurate quantification of selenoproteins in human plasma/serum by isotope dilution ICP-MS: focus on selenoprotein $\mathrm{P}^{\prime \prime}$, J. Anal. At. Spectrom. 31, 1904 (2016). https:// doi.org/10.1039/C6JA00122J

9. A. Tchaikovsky, A. Schoeber, H. Schueff, A. Raab, S. Emin, A. Slany, P. Heffeter, G. Koellensperger and C. Swart, "Quantification of ferritinbound iron in murine samples for Alzheimer's disease studies using species-specific isotope dilution mass spectrometry", Metrologia 57, 042101 (2020). https://doi. org/10.1088/1681-7575/ab8c9f

10. R. Larios, M.E. Del Castillo Busto, D. Garcia-Sar, C. Ward-Deitrich and $\mathrm{H}$. Goenaga-Infante, "Accurate quantification of carboplatin adducts with serum proteins by monolithic chromatography coupled to ICPMS with isotope dilution analysis", J. Anal. At. Spectrom. 34, 729-740 (2019). https://doi.org/10.1039/ C8JA00409A

11. M. Sargent, H. Goenaga-Infante, K. Inagaki, L. Ma, J. Meija, A. Pramann, O. Rienitz, R. Sturgeon, J. Vogl, J. Wang and L. Yang, "The role of ICP-MS in inorganic chemical metrology", Metrologia 56, 034005 (2019). https://doi.org/10.1088/1681-7575/ ab0eac

12. M. Sargent, C. Harrington and R. Harte, Guidelines for Achieving High Accuracy in Isotope Dilution Mass Spectrometry. Royal Society of Chemistry, Cambridge, UK (2002). ISBN 0-85404-418-3, https://doi. org/10.1039/9781847559302 